\title{
Effects of fenofibrate on renal function in patients with type 2 diabetes mellitus: the Fenofibrate Intervention and Event Lowering in Diabetes (FIELD) Study
}

\author{
T. M. E. Davis • R. Ting • J. D. Best • M. W. Donoghoe • \\ P. L. Drury • D. R. Sullivan • A. J. Jenkins • \\ R. L. O'Connell • M. J. Whiting • P. P. Glasziou • \\ R. J. Simes • Y. A. Kesäniemi • V. J. Gebski • R. S. Scott • \\ A. C. Keech • on behalf of the FIELD Study investigators
}

Received: 23 June 2010 / Accepted: 15 September 2010 /Published online: 4 November 2010

(C) Springer-Verlag 2010

\begin{abstract}
Aims/hypothesis Fenofibrate caused an acute, sustained plasma creatinine increase in the Fenofibrate Intervention and Event Lowering in Diabetes (FIELD) and Action to Control Cardiovascular Risk in Diabetes (ACCORD)
\end{abstract}

T. M. E. Davis and R. Ting are joint first authors.

Electronic supplementary material The online version of this article (doi:10.1007/s00125-010-1951-1) contains supplementary material,

which is available to authorised users.

T. M. E. Davis

School of Medicine and Pharmacology, Fremantle Hospital,

University of Western Australia,

Fremantle, WA, Australia

R. Ting $(\bowtie) \cdot$ M. W. Donoghoe $\cdot$ R. L. O’Connell $\cdot$ R. J. Simes

V. J. Gebski $\cdot$ A. C. Keech $(\square)$

The FIELD Study, c/o NHMRC Clinical Trials Centre,

Level 6, Medical Foundation Building, K25,

University of Sydney, NSW 2006, Australia

e-mail: rudee.ting@ctc.usyd.edu.au

A. C. Keech

e-mail: tony@ctc.usyd.edu.au

J. D. Best · A. J. Jenkins

Department of Medicine, St Vincent's Hospital,

University of Melbourne,

Melbourne, Victoria, Australia

\section{P. L. Drury}

Auckland Diabetes Centre, Greenlane Clinical Centre,

Greenlane West,

Auckland, New Zealand

R. Ting $\cdot$ D. R. Sullivan $\cdot$ A. C. Keech

Royal Prince Alfred Hospital,

Sydney, NSW, Australia studies. We assessed fenofibrate's renal effects overall and in a FIELD washout sub-study.

Methods Type 2 diabetic patients $(n=9,795)$ aged 50 to 75 years were randomly assigned to fenofibrate $(n=4,895)$ or placebo $(n=4,900)$ for 5 years, after 6 weeks fenofibrate

M. J. Whiting

Flinders Medical Centre,

Adelaide, SA, Australia

P. P. Glasziou

Centre for Evidence-Based Medicine, University of Oxford,

Oxford, UK

Y. A. Kesäniemi

Institute of Clinical Medicine, Department of Internal Medicine,

University of Oulu,

Oulu, Finland

Y. A. Kesäniemi

Biocenter Oulu, University of Oulu,

Oulu, Finland

Y. A. Kesäniemi

Clinical Research Centre, Oulu University Hospital,

Oulu, Finland

R. S. Scott

Lipid \& Diabetes Research Group, Christchurch Hospital,

Christchurch, New Zealand 
run-in. Albuminuria (urinary albumin/creatinine ratio measured at baseline, year 2 and close-out) and estimated GFR, measured four to six monthly according to the Modification of Diet in Renal Disease Study, were pre-specified endpoints. Plasma creatinine was re-measured 8 weeks after treatment cessation at close-out (washout sub-study, $n=661$ ). Analysis was by intention-to-treat.

Results During fenofibrate run-in, plasma creatinine increased by $10.0 \mu \mathrm{mol} / 1(p<0.001)$, but quickly reversed on placebo assignment. It remained higher on fenofibrate than on placebo, but the chronic rise was slower (1.62 vs $1.89 \mu \mathrm{mol} / 1$ annually, $p=0.01$ ), with less estimated GFR loss (1.19 vs $2.03 \mathrm{ml} \mathrm{min}^{-1} 1.73 \mathrm{~m}^{-2}$ annually, $p<0.001$ ). After washout, estimated GFR had fallen less from baseline on fenofibrate $\left(1.9 \mathrm{ml} \mathrm{min}^{-1} 1.73 \mathrm{~m}^{-2}, p=0.065\right)$ than on placebo $\left(6.9 \mathrm{ml} \mathrm{min}^{-1} 1.73 \mathrm{~m}^{-2}, p<0.001\right)$, sparing $5.0 \mathrm{ml} \mathrm{min}^{-1}$ $1.73 \mathrm{~m}^{-2}$ (95\% CI 2.3-7.7, $\left.p<0.001\right)$. Greater preservation of estimated GFR with fenofibrate was observed with baseline hypertriacylglycerolaemia ( $n=169$ vs 491 without) alone, or combined with low HDL-cholesterol ( $n=140$ vs 520 without) and reductions of $\geq 0.48 \mathrm{mmol} / 1$ in triacylglycerol over the active run-in period (pre-randomisation) ( $n=356$ vs 303 without). Fenofibrate reduced urine albumin concentrations and hence albumin/creatinine ratio by $24 \%$ vs $11 \%$ $(p<0.001$; mean difference 14\% [95\% CI 9-18]; $p<0.001)$, with $14 \%$ less progression and $18 \%$ more albuminuria regression $(p<0.001)$ than in participants on placebo. Endstage renal event frequency was similar $(n=21$ vs $26, p=0.48)$. Conclusions/interpretation Fenofibrate reduced albuminuria and slowed estimated GFR loss over 5 years, despite initially and reversibly increasing plasma creatinine. Fenofibrate may delay albuminuria and GFR impairment in type 2 diabetes patients. Confirmatory studies are merited.

\section{Trial registration ISRCTN64783481}

Funding The study was funded by grants from Laboratoires Fournier SA (Dijon, France; now part of Abbott Pharmaceuticals) and the National Health and Medical Research Council, Australia

Keywords Albuminuria $\cdot$ Creatinine $\cdot$ Diabetes .

Fenofibrate $\cdot$ FIELD $\cdot$ GFR $\cdot$ Nephropathy

Renal impairment

\section{Abbreviations \\ ACCORD}

Action to Control Cardiovascular Risk in Diabetes

ACR

FIELD
Albumin/creatinine ratio

Fenofibrate Intervention and Event

Lowering in Diabetes
MICRO-HOPE Microalbuminuria Cardiovascular, and

Renal Outcomes-Heart Outcomes

Prevention Evaluation

PAI-1 Plasminogen activating inhibitor-1

\section{Introduction}

Two recent large-scale, double-blind, randomised, placebocontrolled clinical trials, Fenofibrate Intervention and Event Lowering in Diabetes (FIELD) and Action to Control Cardiovascular Risk in Diabetes (ACCORD), have shown an early, sustained rise in serum creatinine with fenofibrate in patients with type 2 diabetes $[1,2]$. Given the benefits of reduced cardiovascular events in the subgroups with dyslipidaemia (hypertriacylglycerolaemia and low HDLcholesterol) in both studies and despite negative primary endpoints overall (FIELD: non-fatal myocardial infarction and coronary death; ACCORD: non-fatal myocardial infarction, non-fatal stroke and cardiovascular disease death), it is important to determine the clinical significance of this plasma creatinine rise. In both trials, fenofibrate was generally safe and well tolerated, but with a small increase in rates of pancreatitis and pulmonary embolism in FIELD $[1,2]$. In FIELD, fewer microvascular amputations and less laser-requiring retinopathy occurred in fenofibrate-treated patients (also confirmed in ACCORD-EYE [3]), potentially further broadening the clinical applications of fenofibrate $[4,5]$.

Diabetes is a leading cause of renal dysfunction and endstage renal disease. One in five diabetic patients progresses to end-stage renal disease within 20 years of nephropathy onset [6]. Cardiovascular complications start early in renal disease with even minor renal deterioration being associated with arterial thickening [7]. Current therapies may not arrest renal function decline and there is an urgent need for new targets and interventions [8], as renal failure and associated cardiovascular disease increase mortality rates [9]. Fenofibrate has been shown to decrease albuminuria in a mouse model of type 2 diabetes [10] and in humans $[1,2,11]$. Given potential renal benefit, but the possible safety concerns arising from the rise in plasma creatinine, we carried out a detailed analysis of the effects of long-term fenofibrate treatment on pre-specified renal outcomes during treatment and after drug cessation.

\section{Methods}

Design overview FIELD was a randomised, double-blind placebo controlled trial with the primary outcome of coronary events. Study design and patient characteristics have been published elsewhere [1]. This registered study had ethics 
committee approval in accordance with the Declaration of Helsinki and Good Clinical Practice Guidelines.

Setting and participants Sites $(n=63)$ in Australia, New Zealand and Finland recruited 9,795 type 2 diabetic participants aged 50 to 75 years and with baseline plasma total cholesterol between 3.0 and $6.5 \mathrm{mmol} / \mathrm{l}$, plus total cholesterol/HDL-cholesterol ratio $\geq 4.0$ or plasma triacylglycerol ranging from 1.0 to $5.0 \mathrm{mmol} / \mathrm{l}$, and without need of lipid-lowering drugs. Exclusion criteria were plasma creatinine $>130 \mu \mathrm{mol} / 1$, liver or symptomatic gallbladder disease, or a cardiovascular event within 3 months prior to recruitment. All patients provided written informed consent and then completed a 16 week run-in period comprising 4 weeks of diet, 6 weeks of single-blind placebo and 6 weeks of single-blind fenofibrate. Eligibility was confirmed during run-in independently of adherence or biochemical changes.

Randomisation and interventions Participants with type 2 diabetes $(n=9,795)$ were assigned to fenofibrate $200 \mathrm{mg}$ or placebo daily for 5 years on average. A telephone computer randomisation service using dynamic balancing to stratify patients by prognostic variables was used. All investigators, except the authorised study statistician, were masked to treatment allocation before and after randomisation.

Outcomes and follow-up Pre-specified renal endpoints were: (1) renal function changes; (2) urinary albumin: creatinine ratio (ACR) changes; and (3) end-stage renal disease, defined as plasma creatinine $>400 \mu \mathrm{mol} / \mathrm{l}$, dialysis, transplant or renal disease death. Albuminuria at baseline was defined as: (1) microalbuminuria, i.e. urinary ACR $\geq 2.5$ and $\geq 3.5 \mathrm{mg} / \mathrm{mmol}$ (men and women respectively); and (2) macroalbuminuria, i.e. urinary ACR $>25$ and $>35 \mathrm{mg} / \mathrm{mmol}$ (men and women respectively). This definition was revised to be sex-specific after the study was unmasked. Estimated GFR was by the Modification of Diet in Renal Disease (MDRD) study four-variable formula [12] and categorised as estimated GFR $\geq 90 \mathrm{ml} \mathrm{min}^{-1} 1.73 \mathrm{~m}^{-2} ; 60$ to $<90 \mathrm{ml} \mathrm{min}^{-1} 1.73 \mathrm{~m}^{-2}$; 30 to $<60 \mathrm{ml} \mathrm{min}^{-1} 1.73 \mathrm{~m}^{-2}$; and $<30 \mathrm{ml} \mathrm{min}^{-1} 1.73 \mathrm{~m}^{-2}$. All measurements of albuminuria and creatinine were performed in the central laboratories with regular quality assurance through participation in national schemes. Plasma creatinine was measured by the Jaffe reaction (alkaline picrate-kinetic) on a clinical chemistry analyser (Hitachi 917; Roche Diagnostics, Basel, Switzerland), using calibrators supplied by the manufacturer, with interassay CVs over 5 years of $1.3 \%$ at a concentration of $170 \mu \mathrm{mol} / \mathrm{l}$ and $1.9 \%$ at $600 \mu \mathrm{mol} / \mathrm{l}$. Urinary creatinine was also measured on this analyser after dilution. This method showed excellent agreement with isotope-dilution mass spectrometry and recovered the certified values assigned to the reference material.
Urine albumin was measured by immunonephelometry on an analyser (Array 360; Beckman, Fullerton, CA, USA), with interassay CVs over 5 years of $2.8 \%$ at a concentration of $10 \mathrm{mg} / 1$ and $2.5 \%$ at $50 \mathrm{mg} / 1$.

Although estimated GFR may underestimate true glomerular filtration rate [13], particularly at $>90 \mathrm{ml} \mathrm{m^{-1 }}$ $1.73 \mathrm{~m}^{-2}$, our analyses focused on changes over time and between treatments, rather than absolute values.

Vascular complications were self-reported at screening. Lipids, $\mathrm{HbA}_{1 \mathrm{c}}$, renal function, hepatic function and first morning urinary ACR were measured with annual calibration. Patients were seen at intervals of 4 to 6 months against a background of usual care, and information on treatment toleration and complications was obtained. Urinary albumin and creatinine were measured pre-randomisation (the mean of two first morning samples) and at year 2, year 5 and close-out.

Washout sub-study In analyses pre-specified 6 months prior to unmasking of the study, 661 patients were re-assessed $52 \pm 13$ days after close-out to further evaluate the known fenofibrate-associated changes in plasma creatinine. Given that fenofibrate has a $20 \mathrm{~h}$ elimination half-life, this timepoint minimised any drug withdrawal rebound effect. Substudy patients all gave consent at the main study close-out at participating centres after local ethical approval for the additional washout visit had been granted. They had similar baseline characteristics to non-participants and balanced characteristics by treatment allocation (Electronic supplementary material [ESM] Table 1). At washout-period end, fasting plasma was collected.

Statistical analysis Comparisons of pre-specified baseline variables used $t$ tests or Wilcoxon's rank-sum tests for continuous variables, and $\chi^{2}$ tests for categorical variables. Comparisons of changes in estimated GFR between groups used $t$ tests within each subgroup. Interaction tests used a linear model, with estimated GFR change as the dependent variable and $p$ value for interaction based on the likelihood ratio test for interaction between subgroup and treatment variables. Urinary ACR (log transformed) and estimated GFR analyses were similar. Predictors of baseline estimated GFR, and changes in plasma creatinine, estimated GFR and urinary ACR were determined using linear models and stepwise variable selection from a predefined list. Only the analyses relating to changes in estimated GFR from baseline to the end of the washout period according to baseline characteristics and changes in biomarkers during active run-in were post hoc, in that the associations between the prespecified variables and the pre-specified outcomes were not expressly stated in the statistical analysis plan. Numbers needed to treat were calculated by transforming the absolute risk reduction. Two-sided $p$ values were significant at $p<0.05$ and were unadjusted for multiple comparisons. 
Fig. 1 Trial profile of renal study. eGFR, estimated GFR

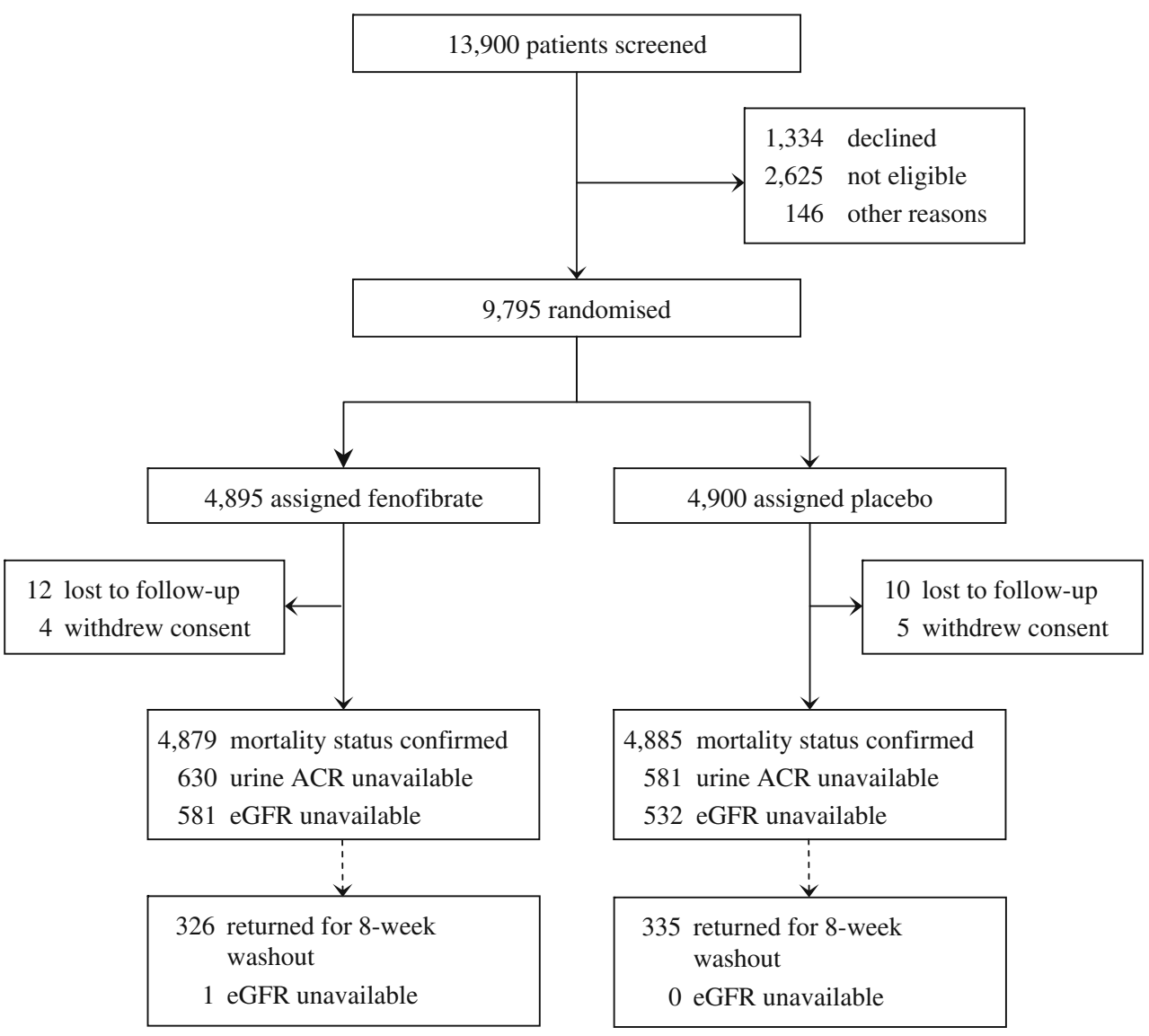

\section{Results}

Of 13,900 screened patients, 9,795 were randomised to fenofibrate $(n=4,895)$ or placebo $(n=4,900$; Fig. 1$)$. The groups were well matched including for renal function, blood pressure and glycaemia (Table 1). Approximately 20\% reported microvascular complications. At baseline, 2,508 (26\%) patients had increased urinary ACR and 5,737 (59\%) had estimated GFR of 30 to $89 \mathrm{ml} \mathrm{min}^{-1} 1.73 \mathrm{~m}^{-2}$. Few had macroalbuminuria $(404,4 \%)$ or estimated GFR of 30 to $<60 \mathrm{ml} \mathrm{min}{ }^{-1} 1.73 \mathrm{~m}^{-2}(519,5 \%)$. Almost $80 \%$ with estimated GFR $<90 \mathrm{ml} \mathrm{min}^{-1} 1.73 \mathrm{~m}^{-2}$ had normal ACR, and $40 \%$ with raised ACR had estimated GFR $\geq 90 \mathrm{ml} \mathrm{min}^{-1}$ $1.73 \mathrm{~m}^{-2}$ (ESM Table 2).

Fenofibrate effects on plasma creatinine and estimated GFR Mean plasma creatinine increased $10.0 \mu \mathrm{mol} / \mathrm{l}$ during the pre-randomisation 6 week fenofibrate run-in period. In those subsequently randomised to placebo, levels at the next visit (4 months) fell back to baseline levels, but then increased by $1.7 \mu \mathrm{mol} / \mathrm{l}$ per year (Fig. $2 \mathrm{a}$ ). In the same group, estimated GFR fell proportionately and below $90 \mathrm{ml} \mathrm{min}{ }^{-1}$ $1.73 \mathrm{~m}^{-2}$ at a rate of approximately $5 \%$ per annum. Baseline risk factors for greater plasma creatinine rise and estimated GFR loss during placebo treatment over 5 years included elevated urinary ACR, older age, lower HDL-cholesterol and higher $\mathrm{HbA}_{1 \mathrm{c}}(p<0.001$ for all). Placebo patients requiring renin-angiotensin system blockers at entry also had greater estimated GFR loss than other participants $(p=0.004)$, possibly representing indication bias.

In participants allocated fenofibrate, plasma creatinine remained 10 to $12 \mu \mathrm{mol} / 1$ higher than placebo $(p<0.001$; Fig. 2a), with an apparently greater fall in estimated GFR ( 87.6 to 70.5 vs 87.8 to $79.9 \mathrm{ml} \mathrm{min}{ }^{-1} 1.73 \mathrm{~m}^{-2}$ ) from prerun-in (baseline) to close-out. However, the long-term plasma creatinine rise (4 months to close-out) was smaller with fenofibrate than with placebo, both overall $(7.9 \mu \mathrm{mol} / \mathrm{l}$ vs $9.2 \mu \mathrm{mol} / 1, p=0.01$; Fig. $2 \mathrm{a}$ ), and within each subgroup of baseline estimated GFR (ESM Fig. 1). This was paralleled by slower estimated GFR loss (5.8 vs $9.9 \mathrm{ml} \mathrm{min}^{-1} 1.73 \mathrm{~m}^{-2}$, respectively, $p<0.001$; Fig. $2 \mathrm{~b}$ ). The mean annual rates of plasma creatinine rise and estimated GFR decline were 1.62 vs $1.89 \mu \mathrm{mol} / \mathrm{l}$ and $1.19 \mathrm{vs} 2.03 \mathrm{ml} \mathrm{min}^{-1} 1.73 \mathrm{~m}^{-2}$ respectively for patients on fenofibrate vs those on placebo.

In the 661 washout sub-study participants, plasma creatinine changes (baseline to closeout) were comparable to those in the whole cohort, but levels at 8 weeks after withdrawal of study treatment were significantly lower in participants allocated fenofibrate than in those on placebo $(p<0.001$; Fig. 3a). This reflected a 5 -year fall in estimated GFR of $1.9 \mathrm{ml} \mathrm{min}^{-1} 1.73 \mathrm{~m}^{-2}$ in the fenofibrate group (from 89.2 to 87.3 ; $p=0.07)$ vs $6.9 \mathrm{ml} \mathrm{min}^{-1} 1.73 \mathrm{~m}^{-2}$ in the placebo group 
Table 1 Baseline characteristics of all patients by treatment
Values are mean (SD) or median (interquartile range), unless indicated otherwise

$\mathrm{ARB}$, angiotensin-receptor blocker; eGFR, estimated GFR

${ }^{\mathrm{a}}$ Self-reported at baseline

${ }^{\mathrm{b}}$ Marked dyslipidaemia: low HDLcholesterol (men $<1.03 \mathrm{mmol} / \mathrm{l}$, women $<1.29 \mathrm{mmol} / \mathrm{l})$ with high triacylglycerol $(\geq 2.3 \mathrm{mmol} / \mathrm{l})$

${ }^{\mathrm{c}}$ Alone or in combination

\begin{tabular}{|c|c|c|}
\hline \multirow[t]{2}{*}{ Characteristic } & \multicolumn{2}{|l|}{ Treatment } \\
\hline & Placebo & Fenofibrate \\
\hline$n$ & 4,900 & 4,895 \\
\hline \multicolumn{3}{|l|}{ General } \\
\hline Male, $n(\%)$ & $3,067(62.6)$ & $3,071(62.7)$ \\
\hline Age at visit 1 (years) & $62.23(6.91)$ & $62.23(6.83)$ \\
\hline Duration of diabetes (years) & $5.00(2.00-10.00)$ & $5.00(2.00-10.00)$ \\
\hline \multicolumn{3}{|l|}{ Clinical history } \\
\hline Nephropathy, $n(\%)^{\mathrm{a}}$ & $135(2.8)$ & $144(2.9)$ \\
\hline \multicolumn{3}{|l|}{ Laboratory data } \\
\hline LDL-cholesterol (mmol/1) & $3.07(0.66)$ & $3.07(0.64)$ \\
\hline HDL-cholesterol (mmol/l) & $1.10(0.26)$ & $1.10(0.26)$ \\
\hline Triacylglycerol (mmol/l) & $1.73(1.34-2.30)$ & $1.74(1.35-2.34)$ \\
\hline Marked dyslipidaemia, $n(\%)^{\mathrm{b}}$ & $970(19.8)$ & $1,044(21.3)$ \\
\hline $\mathrm{HbA}_{1 \mathrm{c}}(\%)$ & $6.85(6.10-7.75)$ & $6.85(6.05-7.80)$ \\
\hline Plasma creatinine $(\mu \mathrm{mol} / \mathrm{l})$ & $77.40(15.66)$ & $77.73(15.91)$ \\
\hline Urine ACR (mg/mmol) & $1.10(0.60-2.90)$ & $1.15(0.60-3.00)$ \\
\hline Normoalbuminuria, $n(\%)$ & $3,643(74.5)$ & $3,617(74.1)$ \\
\hline Microalbuminuria, $n(\%)$ & $1,040(21.3)$ & $1,064(21.8)$ \\
\hline Macroalbuminuria, $n(\%)$ & $204(4.2)$ & $200(4.1)$ \\
\hline eGFR (ml min $\left.{ }^{-1} 1.73 \mathrm{~m}^{-2}\right)$ & $87.8(18.3)$ & $87.6(18.5)$ \\
\hline eGFR <60, $n(\%)$ & $224(4.6)$ & $295(6.0)$ \\
\hline eGFR $60-<90, n(\%)$ & $2,657(54.2)$ & $2,561(52.3)$ \\
\hline $\mathrm{eGFR} \geq 90, n(\%)$ & $2,019(41.2)$ & $2,039(41.7)$ \\
\hline \multicolumn{3}{|l|}{ Medication } \\
\hline ACE inhibitor, $n(\%)$ & $1,653(33.7)$ & $1,628(33.3)$ \\
\hline $\mathrm{ARB}, n(\%)$ & $253(5.2)$ & $269(5.5)$ \\
\hline Any insulin, $n(\%)^{\mathrm{c}}$ & $672(13.7)$ & $674(13.8)$ \\
\hline
\end{tabular}

(from 87.5 to $80.6 ; p<0.001$ ), such that fenofibrate spared an average of $5.0 \mathrm{ml} \mathrm{min}-1.73 \mathrm{~m}^{-2}(p<0.001)$ or $\sim 1 \mathrm{ml} \mathrm{min}^{-1}$ $1.73 \mathrm{~m}^{-2}$ annually (Fig. 3b).

Significant renal function decline was seen in all washout subgroups receiving placebo (Fig. 4). Independently statistically significant estimated GFR preservation with fenofibrate occurred in most subgroups. Evidence of greater benefit was present in those with baseline hypertriacylglycerolaemia $(\geq 2.3 \mathrm{mmol} / \mathrm{l})$ or dyslipidaemia compared with those without ( $p=0.03$ for interaction, for both; Fig. 4). Benefit also differed by treatment response according to the extent of plasma triacylglycerol lowering with fenofibrate (during active run-in), being observed continuously $(p=0.002)$ and categorically ( $p=0.02$ for interaction; ESM Table 3), but independently of all other variables (including other baseline lipids, blood pressure and renin-angiotensin system blocker use; Fig. 4) and on-study commencement of statins or reninangiotensin system blockers (data not shown).

Albuminuria and effects of fenofibrate In both groups, urinary ACR fell over 5 years (Fig. 2c). Determinants of less than average improvement were low urinary ACR or high plasma creatinine at baseline, current smoking, longer diabetes duration and older age. Other predictors (also associated with decline in estimated GFR) included increased plasma homocysteine, $\mathrm{HbA}_{1 \mathrm{c}}$ and renin-angiotensin-system blocker use $(p<0.05$ for all). The fall was greater in participants on fenofibrate $(23.7 \%$ vs $11.5 \%$; mean difference $13.9 \%$ [95\% CI 9.2-18.3]; $p<0.001)$. A fall in urinary creatinine concentration was observed with fenofibrate, but this was exceeded by the decrease in urinary albumin concentration, reducing the ratio. This equated to a $0.2 \mathrm{mg} / \mathrm{mmol}$ difference in mean ACR at close-out (fenofibrate 1.1 vs placebo $1.3 \mathrm{mg} / \mathrm{mmol}$ ), reflecting $11.7,1.0$ and $0.1 \mathrm{mg} / \mathrm{mmol}$ differences among macro-, microand normoalbuminuric participants respectively (ESM Fig. 2). A shift across categories occurred, favouring fenofibrate, with $14 \%$ less progression (11.1\% vs $12.9 \%$ ) and $18 \%$ more regression ( $11.2 \%$ vs $9.5 \% ; p<0.001$; ESM Table 4$)$. These benefits were similar for all subgroups (including reninangiotensin system blocker use), except sex, with larger effects among men than women (post hoc analysis, data not shown). 

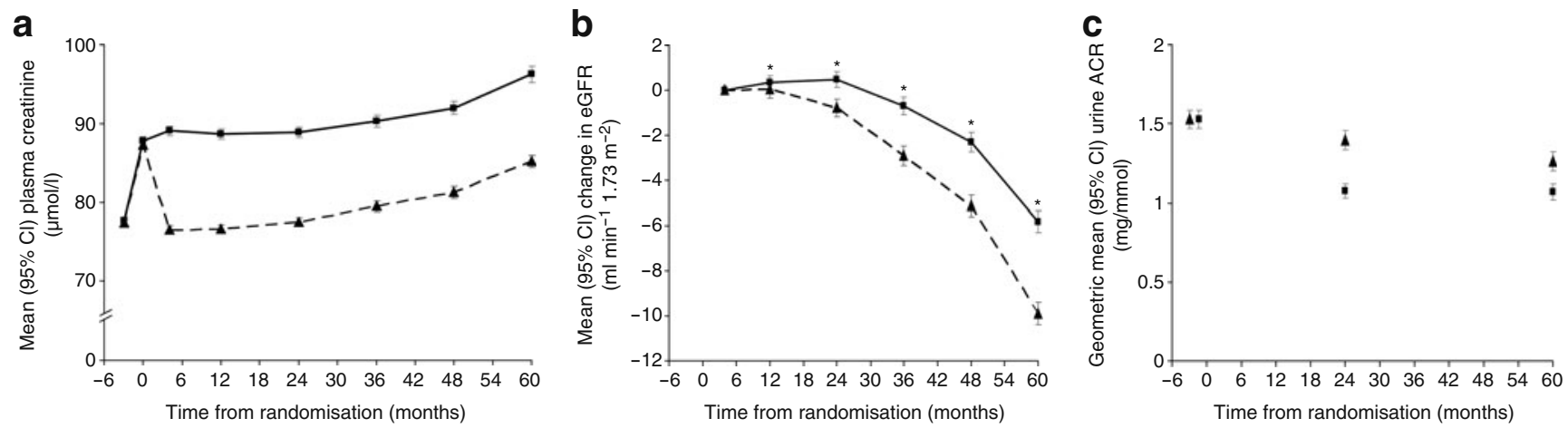

Fig. 2 Changes among all 9,795 patients over 5 years follow-up in (a) plasma creatinine, (b) estimated GFR and (c) ACR in the fenofibrate (squares) and placebo (triangles) groups. Changes are shown from screening for plasma creatinine and urinary ACR, and from 4 months for estimated GFR. *Values for estimated GFR in patients while on fenofibrate may be unreliable and may underestimate true GFR
More advanced renal endpoints Plasma creatinine doubling was more common in participants on fenofibrate than in those on placebo $(3.0 \%$ vs $1.8 \%, p<0.001)$, but was often transient and seldom led to withdrawal ( 7 vs $2, p=0.1$ ). End-stage renal disease (creatinine $>400 \mu \mathrm{mol} / 1$ [ $n=6$ vs 3], dialysis $[n=16$ vs 21$]$, renal transplant $[n=0$ vs 0$]$ and/or renal death $[n=1$ vs 4$]$ ) did not differ between groups (21 in fenofibrate group vs 26 for placebo, $p=0.48$; Table 2). The reduction in the secondary endpoint of total cardiovascular events was no smaller in participants with larger creatinine increases and on fenofibrate than in others (ESM Fig. 3).

\section{Discussion}

Diabetes, a leading cause of renal damage and end-stage renal disease $[6,14]$, is associated with increased mortality rates and substantial personal and economic cost [14]. In 2007 one US diabetic patient started dialysis every $11 \mathrm{~min}$, with associated total costs of US\$33 billion [14]. An ageing population and increasing diabetes prevalence will increase the burden of diabetic renal disease [8]. Despite multifactorial treatment in the STENO-2 trial, renal disease still developed in $25 \%$ of patients over 13 years [15]. We demonstrate here that fenofibrate reduces albuminuria progression and decline of estimated GFR in type 2 diabetes patients, despite a small initial plasma creatinine rise. The greater decrease in albuminuria with fenofibrate than with placebo was independent of baseline characteristics (except sex), while estimated GFR preservation was greater in patients with baseline dyslipidaemia and greater fenofibrate-related plasma triacylglycerol reduction. Therefore, different underlying mechanisms are likely.

With regard to renal filtration function, our results imply two fenofibrate-specific effects: an initial, well-recognised plasma creatinine rise [16] and longer term estimated GFR preservation. The latter is most evident after treatment withdrawal. The early plasma creatinine change does not represent true nephrotoxicity, since there was rapid reversion to pre-treatment levels in those subsequently allocated placebo after the run-in phase. In addition, plasma creatinine after washout at 5 years in those allocated fenofibrate fell below that of the placebo-treated patients.
Fig. 3 Changes among 661 participants in the washout substudy from baseline to 8 weeks after study close for (a) mean plasma creatinine in the fenofibrate group (continuous line) and placebo group (dashed line), and (b) for estimated GFR at baseline (white) and after washout (black). Values are mean (95\% CI); ${ }^{\dagger} p=0.0003$; ${ }^{\ddagger} p=0.065 ;{ }^{\S} p<0.0001$ a

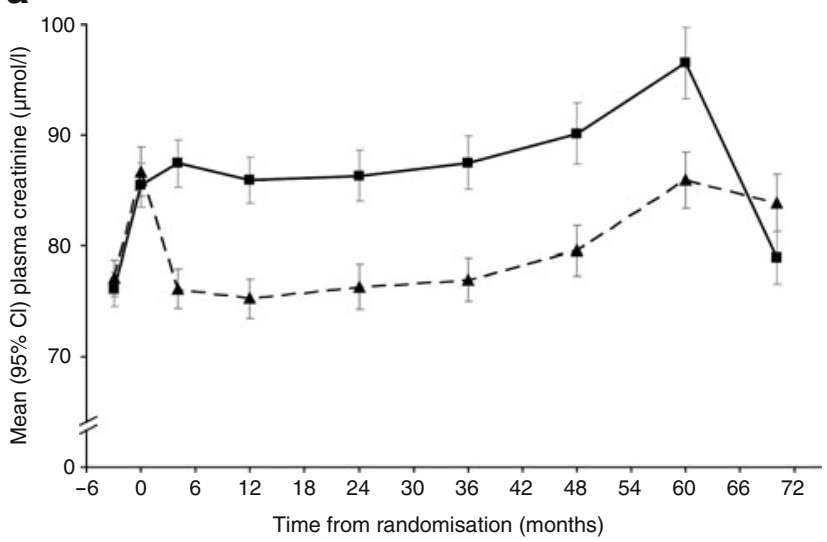

b

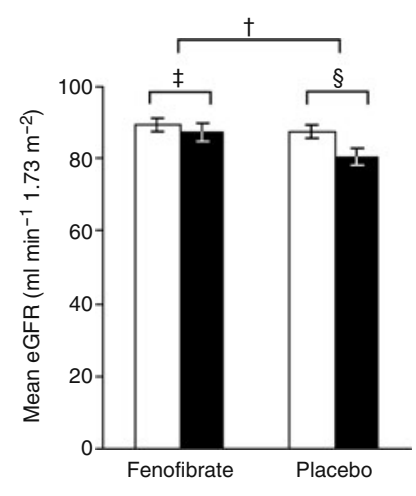


Fig. 4 Forest plot of the change in estimated GFR by treatment group (washout minus baseline, $\mathrm{ml} \mathrm{min}^{-1} 1.73 \mathrm{~m}^{-2}$ ) for baseline subgroups. ${ }^{\mathrm{a}}$ Independently significant change from baseline $(p<0.05)$; subgroups are based on prespecified classifications based on clinical relevance or approximate medians. ${ }^{\mathrm{b}}$ Low HDLcholesterol: men $<1.03 \mathrm{mmol} / \mathrm{l}$, women $<1.29 \mathrm{mmol} / 1$. ${ }^{\mathrm{c}}$ Treatment $\times$ subgroup interactions were significant only for triacylglycerol $(p=0.03)$ and marked dyslipidaemia $(p=0.03)$. ${ }^{\mathrm{d}}$ Marked dyslipidaemia: triacylglycerol $\geq 2.3 \mathrm{mmol} / 1$ and low HDL-cholesterol.

${ }^{\mathrm{e}}$ Microalbuminuria was defined as urinary ACR $\geq 2.5 \mathrm{mg} / \mathrm{mmol}$ (men) and $\geq 3.5 \mathrm{mg} / \mathrm{mmol}$ (women); macroalbuminuria as urinary ACR $>25 \mathrm{mg} / \mathrm{mmol}$ (men) or $>35 \mathrm{mg} / \mathrm{mmol}$ (women) (revised sex-specific cut-points) [45]. ARB, angiotensin-receptor blocker. eGFR, estimated GFR

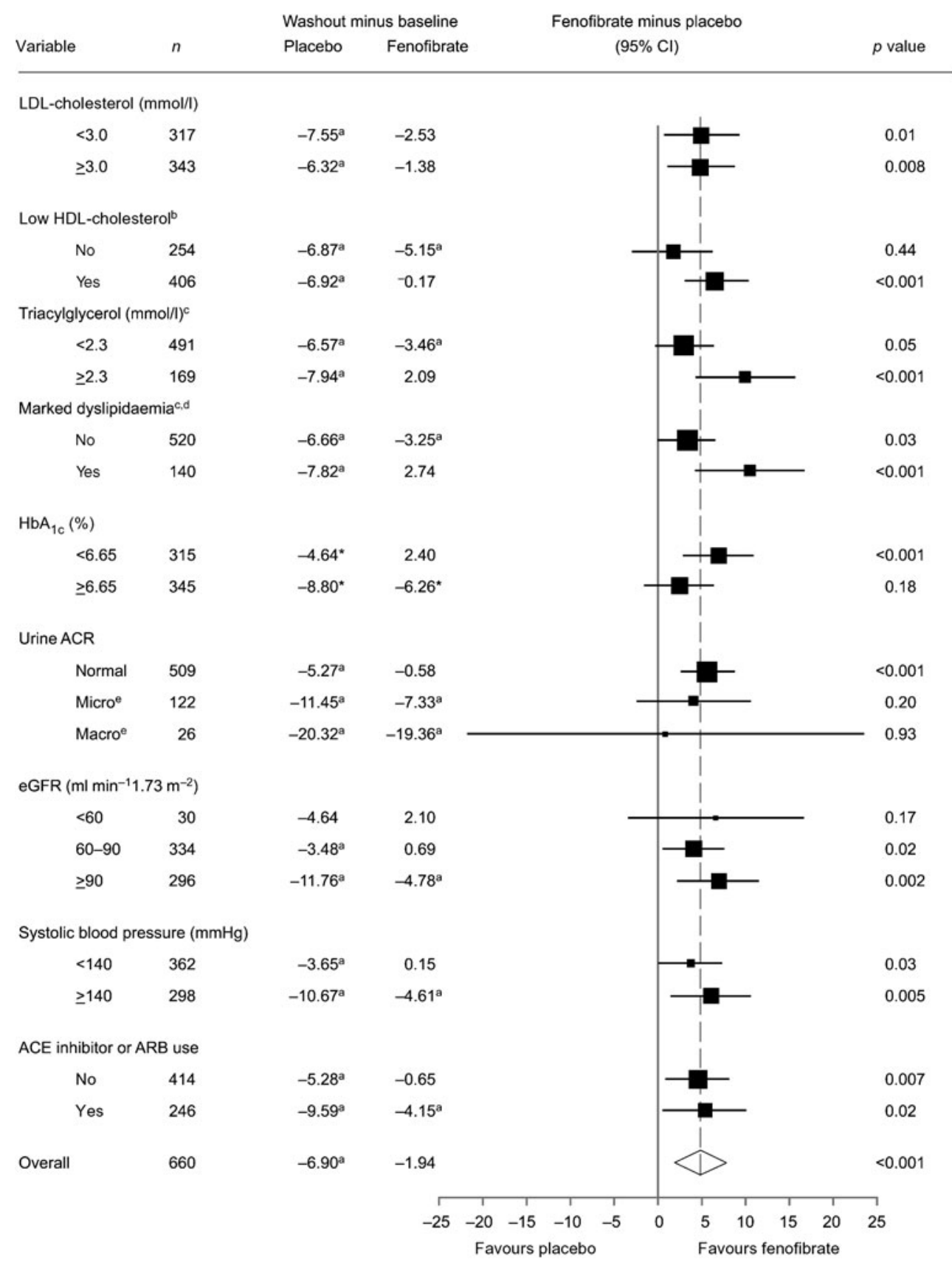

linked with reduction in albuminuria, as well as histopathological improvements, e.g. reduced glomerular hypertrophy and reduced mesangial expansion [10]. The ACCORD Lipid trial, which examined patients on a background of statin treatment and high use of renin-angiotensin system blockade, recently confirmed our finding that fenofibrate caused an acute plasma creatinine rise and long-term reduction of micro- and macroalbuminuria, with no adverse effect on end-stage renal disease compared with placebo $[1,2]$. A Helsinki sub-study examined renal function in 170 Finnish participants in FIELD [21]. The investigators were unable to demonstrate a reduction in albuminuria with fenofibrate, despite the highly significant findings in the 
Table 2 Pre-specified end-stage renal events by treatment

\begin{tabular}{|c|c|c|c|c|c|c|}
\hline \multirow[t]{2}{*}{ Variable } & \multicolumn{2}{|c|}{ Placebo } & \multicolumn{2}{|c|}{ Fenofibrate } & \multicolumn{2}{|l|}{ Total } \\
\hline & $n^{\mathrm{a}}$ & $\%$ & $n^{\mathrm{a}}$ & $\%$ & $n^{\mathrm{a}}$ & $\%$ \\
\hline Participants & 4,900 & 100 & 4,895 & 100 & 9,795 & 100 \\
\hline \multicolumn{7}{|l|}{ Event } \\
\hline Plasma creatinine $>400 \mu \mathrm{mol} / 1$ & 3 & $(0.1)$ & 6 & $(0.1)$ & 9 & $(0.1)$ \\
\hline Renal replacement therapy & 21 & $(0.4)$ & 16 & $(0.3)$ & 37 & $(0.4)$ \\
\hline Renal transplant & 0 & $(0.0)$ & 0 & $(0.0)$ & 0 & $(0.0)$ \\
\hline Death from renal disease & 4 & $(0.1)$ & 1 & $(0.0)$ & 5 & $(0.1)$ \\
\hline Total patients with ESRD & 26 & $(0.5)$ & 21 & $(0.4)$ & 47 & $(0.5)$ \\
\hline Doubling of serum creatinine & 90 & $(1.8)$ & 148 & $(3.0)$ & 238 & $(2.4)$ \\
\hline Doubling of serum creatinine or $\mathrm{ESRD}^{\mathrm{b}, \mathrm{c}}$ & 103 & $(2.1)$ & 152 & $(3.1)$ & 255 & $(2.6)$ \\
\hline Doubling of serum creatinine or ESRD ${ }^{\mathrm{b}, \mathrm{d}}$ & 105 & $(2.1)$ & 152 & $(3.1)$ & 257 & $(2.6)$ \\
\hline
\end{tabular}

whole FIELD trial and the ACCORD trial, presumably due to the small sample size. They also found a greater fall in estimated GFR in patients allocated fenofibrate compared with placebo, in keeping with our on-study findings. However, the study had no washout phase and was therefore not able to unmask the underlying GFR preservation demonstrated now by us.

The cystatin $\mathrm{C}$ elevation reported in the Helsinki substudy may indicate altered glomerular function, but might also arise from other clinical and pharmacological factors $[22,23]$. The reasons behind the initial plasma creatinine rise associated with fenofibrate have yet to be fully ascertained. The rise appears to be partly due to a decrease in creatinine clearance $[16,21]$ without reduced inulinderived GFR $[16,24]$, raising the possibility of interference with the active secretion pathway for creatinine in the proximal tubule. In support of this possibility we observed significantly greater increases in plasma creatinine in response to fenofibrate in FIELD patients receiving cimetidine (a well recognised inhibitor of this tubular secretion) than those seen in others $(p=0.002)$. Based on our data, there is no evidence of permanent renal injury. Ansquer et al. hypothesised that reduced tubular secretion could have accounted for the decrease in creatinine clearance they observed, although tubular function assessed by retinol binding protein levels was not changed significantly [16]. Hottelart et al. demonstrated increased creatinuria with no fall in creatinine clearance [25]. Fenofibrate effects on renal plasma flow in these two studies were also contrasting [16, 25], but both concluded there was no loss of glomerular function. While this is encouraging, further research is warranted. Hottelart et al. proposed that an endogenous source of creatinine, presumably from muscle, could augment serum creatinine during fenofibrate treatment [25]. In FIELD, plasma creatine phosphokinase rose $2.4 \%$ in those on fenofibrate vs $0.5 \%$ in those on placebo ( $p=0.06$ for difference) over 5 years of follow-up, while increases in plasma creatinine correlated weakly with increases in creatine phosphokinase (after 6 weeks run-in, $r=0.09$, $p<0.001$; at 5 years, $r=0.14, p<0.001)$, suggesting fenofibrate-associated increased muscle turnover may have contributed in part to the creatininaemia. Thus various hypotheses on the cause of the acute and sustained creatinine elevation due to fenofibrate are possible: (1) increased muscle production of creatine; (2) changes in active tubular creatinine secretion; (3) reduced glomerular function; and (4) altered renal plasma flow. Some or all of these may apply. Regardless of the physiology underlying the increase in creatinine, it is definitely reversible and appears to be a separate process from the underlying renal preservation which is 'masked' during active treatment.

The pattern of early plasma creatinine rise and subsequent attenuation of the rate of renal decline with fenofibrate therapy is similar to that seen with ACE inhibitors, albeit through different mechanisms. ACE inhibitors increase creatinine through a true reduction in GFR secondary to reduced intraglomerular pressure, but are reno-protective because they decrease glomerular hyperfiltration and have anti-fibrogenic effects [26, 27]. The underlying reno-protective mechanisms of fenofibrate remain to be fully elucidated (ESM Table 5).

Hypertriacylglycerolaemia and the degree to which it is reduced were the only characteristics that predicted greater than average preservation of estimated GFR with fenofibrate. As statin trials have not consistently shown renoprotective effects, despite moderate triacylglycerol reduction [28-30], the benefits of fenofibrate may not be solely lipid-mediated. This may reflect antioxidant and antiinflammatory effects [31]. Triacylglycerols have been linked to nephropathy through mesangial cell uptake of very low-density lipoprotein (VLDL), inducing foam cell formation [32] and through VLDL induction of plasminogen activating inhibitor-1 (PAI-1) with upregulated coagulation and intra-renal microthrombi [33]. HDL does not 
greatly alter PAI-1 release [34], but has been shown in animals and humans to be reno-protective [35], perhaps through suppression of inflammatory cell adhesion molecules, anti-oxidant effects and reverse cholesterol transport $[36,37]$. Peroxisome-proliferator-alpha receptor agonists such as fenofibrate may have potential benefits through most of these mechanisms [31]. The observed relationships between both hypertriacylglycerolaemia and dyslipidaemia and renal function in the FIELD study may also reflect insulin resistance which is a risk factor for renal dysfunction [38]. The arguably small absolute fall in estimated GFR in the placebo group ( $8 \%$ over 5 years) may reflect good control of blood pressure, glycaemia and dyslipidaemia [39]. However, based on the placebo rate of estimated GFR loss over 5 years, fenofibrate's estimated GFR benefit would be the equivalent of 3.6 kidney-years saved. This protection was observed across most subgroups and was irrespective of baseline or on-study commencement of renin-angiotensin system blockers. As the subsequent initiation of renin-angiotensin system blockers was higher in the placebo arm (30.1\% vs $25.3 \%$ in fenofibrate group, $p<0.001$ ), the reno-protection provided by fenobrate may have been underestimated. Similarly, commencement of other lipid medications, including statins, was greater in placebo-allocated patients $(36.2 \%$ vs $19.3 \%$ in fenofibrate group) and did not influence benefit. Fenofibrate benefits were independent of blood pressure despite an associated lowering of systolic BP by approximately $2 \mathrm{mmHg}$.

Three-quarters of FIELD placebo-treated patients were normoalbuminuric at baseline, with $2.8 \%$ developing micro- or macroalbuminuria each year, which is similar to progression rates in the UK Prospective Diabetes Study $(2.5 \%)$ and the Microalbuminuria, Cardiovascular, and Renal Outcomes-Heart Outcomes Prevention Evaluation (MICRO-HOPE) Study (2.2\%) [40, 41]. Improvement in urinary ACR with fenofibrate was twofold greater than with placebo. Implications for cardiovascular protection may be relatively small for normoalbuminuric patients, but are greater for those with macroalbuminuria (ESM Fig. 2) [9], in whom the $11 \mathrm{mg} / \mathrm{mmol}$ difference in mean ACR would represent a $1 \%$ lower absolute 5 year risk of cardiovascular disease, based on a diabetes-specific risk calculator [42].

Our study has limitations. The washout sub-study was relatively small, but nevertheless adequately powered for the outcomes examined and with highly statistically significant results obtained. A larger study might have revealed other subgroups likely to benefit more from fenofibrate. Renal assessment through inulin and $p$-amino hippurate clearance is impractical in large trials and estimated GFR has limitations [13]. Nevertheless, comparative temporal within-patient changes in estimated GFR and particularly plasma creatinine in our large sample are likely to remain robust [43]. Moreover, very few participants withdrew due to plasma creatinine rises and, to our knowledge, the washout study is the first of its type among agents with renal effects. Re-analysis of our data using an estimated GFR formula (EPI-CKD) with greater accuracy at higher estimated GFR levels [44] did not alter our conclusions (slightly smaller but still significant benefit [data not shown]). Serum cystatin C, an indicator of renal dysfunction in type 2 diabetes, also modestly increases in patients on fenofibrate without any change in inulin clearance [16] and reverts shortly after drug withdrawal (J.-C. Ansquer, Laboratoires Fournier SA, Dijon, France, personal communication), but has not yet been measured in FIELD. A very similar rise in serum cystatin $C$ was also demonstrated in the Helsinki sub-study [21], but measurements were not repeated after treatment withdrawal. Additional FIELD analyses will facilitate understanding of why fenofibrate may reversibly increase cystatin $C$ [16]. Urinary ACR was measured in FIELD less often than plasma creatinine, but the fenofibrate-associated changes were nevertheless highly significant. Regression to the mean is unlikely to play a major role in these data, as there was no entry criterion related to albuminuria and baseline measures of albuminuria and estimated GFR were both derived as the mean of two pre-randomisation values. One strength of the FIELD study is that it is one of the largest type 2 diabetes randomised trials examining pre-specified renal outcomes. We incorporated variables available in a usual-care context to ensure relevance to clinical practice.

In FIELD, there was evidence that fenofibrate had significant beneficial effects on laser treatment for diabetic retinopathy [4] and lower limb amputations, including in instances regarded as having a predominantly microvascular aetiology [5]. The protective effect of fenofibrate on these two microvascular complications of diabetes is consistent with the present findings of a slowing of the progression of diabetic microvascular renal disease. The number needed to treat with fenofibrate to prevent one patient progressing to micro- or macroalbuminuria is 54 . This compares favourably with 66 to 100 for ACE inhibitor use in MICRO-HOPE [41]. In the FIELD study, the number needed to treat to prevent one patient worsening by at least one estimated GFR grouping $\left(<30,30\right.$ to $<60,60$ to $<90$ or $\geq 90 \mathrm{ml} \mathrm{min}^{-1}$ $1.73 \mathrm{~m}^{-2}$ ) was 25 and only 10 for dyslipidaemic patients. By comparison, the numbers needed to treat to prevent one cardiovascular event overall and in dyslipidaemic FIELD patients are 70 and 23 respectively; for ocular laser therapy overall and in patients with existent retinopathy, the numbers needed to treat are 90 and 17 respectively, and for amputation prevention overall and in those with prior foot ulcer and albuminuria, they are 197 and 25 respectively. Because of greater cardiovascular therapy and statin uptake in the placebo group, these may be underestimates. 
In conclusion, we demonstrated in pre-specified analyses that fenofibrate reduces albuminuria progression and may reduce loss of renal function. This appears to be independent of, and therefore additive to renin-angiotensin system blockade and glycaemic control. We found no evidence that the initial plasma creatinine rise represented true renal injury, a finding that has important implications for clinical care. The size and consistency of the estimated GFR and albuminuria benefits support use of fenofibrate in type 2 diabetes to reduce renal morbidity, especially in patients with dyslipidaemia. Confirmatory studies are merited.

Acknowledgements The study was designed by an independent Management Committee and coordinated by the National Health and Medical Research Council (NHMRC) Clinical Trials Centre, University of Sydney, NSW, Australia. The FIELD study was supported by grants from Laboratoires Fournier, Dijon, France (now part of Abbott Pharmaceuticals) and the NHMRC of Australia (Unit grant, Project grant and Fellowships to A. C. Keech and R. J. Simes). Two non-voting representatives of the main sponsor attended meetings of the Management Committee. Study sponsors had no role in data collection or analysis. The authors had full access to all study data. T. M. E. Davis is supported by an NHMRC Practitioner Fellowship. R. Ting is supported by a University of Sydney Clinical Trials Centre scholarship. We thank the National Heart Foundation, Australia, Diabetes Australia, Diabetes New Zealand and the Finnish Diabetes Association for endorsing the study. We also thank R. Pike (NHMRC Clinical Trials Centre, University of Sydney, NSW, Australia) for her assistance with preparation of this manuscript and J.-C. Ansquer for helpful comments. Thanks also go to the many patients and investigators who participated in the FIELD Study.

Duality of interest Some authors have been reimbursed by the pharmaceutical industry for the costs of participating in scientific meetings, contributing to advisory boards, or doing other research: T. M. E. Davis (sanofi-aventis, Novartis, NovoNordisk, Merck Sharpe \& Dohme, Bristol-Myer Squibb, Solvay, GlaxoSmithKline, Pfizer), R. Ting (Solvay (now Abbott), Servier), P. L. Drury (lecture fees from Eli Lilly), D. R. Sullivan (Solvay (now Abbott), AstraZeneca, Merck Sharpe \& Dohme, Pfizer, Schering Plough), A. J. Jenkins (Medtronic, Merck, Pfizer, sanofi-aventis, Solvay (now Abbott)), R. J. Simes (research grant to University of Sydney from Laboratoires Fournier SA), Y. A. Kesäniemi (research grant from Merck/Schering Plough, Novartis and Fournier; honoraria and/or cost reimbursement for scientific meetings participation from Fournier and Merck/Schering Plough; support for participating in advisory board meetings for Merck/ Schering Plough and AstraZeneca) and A. C. Keech (Merck, BristolMyer Squibb, Abbott, Banyu, Eli Lilly, Novartis, AstraZeneca, Solvay (now Abbott), Roche Diagnostics, Novo Nordisk, Roche, and research grant to University of Sydney from Laboratoires Fournier SA). Y. A. Kesäniemi holds stocks in Orion Pharma. All other authors declare that there is no duality of interest associated with this manuscript.

\section{References}

1. The FIELD Study Investigators (2005) Effects of long-term fenofibrate therapy on cardiovascular events in 9795 people with type 2 diabetes mellitus (the FIELD Study): randomised controlled trial. Lancet 366:1849-1861

2. The ACCORD Study Group (2010) Effects of combination lipid therapy in type 2 diabetes mellitus. N Engl J Med 362:1563-1574
3. The ACCORD Study Group and ACCORD Eye Study Group (2010) Effects of medical therapies on retinopathy progression in type 2 diabetes. N Engl J Med 363:233-244

4. Keech AC, Mitchell P, Summanen PA et al (2007) Effect of fenofibrate on the need for laser treatment for diabetic retinopathy (FIELD Study): a randomised controlled trial. Lancet 370:16871697

5. Rajamani K, Colman PG, Li LP et al (2009) Effect of fenofibrate on amputation events in people with type 2 diabetes mellitus (FIELD Study): a prespecified analysis of a randomised controlled trial. Lancet 373:1780-1788

6. American Diabetes Association (2003) Diabetic nephropathy. Position statement. Diabetes Care 26:S94-S98

7. Kastarinen H, Ukkola O, Kesaniemi YA (2009) Glomerular filtration rate is related to carotid intima-media thickness in middle-aged adults. Nephrol Dial Transplant 24:2767-2772

8. Cooper ME, Jandeleit-Dahm K, Thomas MC (2005) Targets to retard the progression of diabetic nephropathy. Kidney Int 68:1439-1445

9. Drury P, Ting R, Zannino D et al. (2010) Estimated glomerular filtration rate and albuminuria are independent predictors of cardiovascular events and death in type 2 diabetes mellitus: the Fenofibrate Intervention and Event Lowering in Diabetes (FIELD) Study. Diabetologia. doi:10.1007/s00125-010-1854-1

10. Park CW, Zhang Y, Zhang X et al (2006) PPARalpha agonist fenofibrate improves diabetic nephropathy in $\mathrm{db} / \mathrm{db}$ mice. Kidney Int 69:1511-1517

11. Ansquer J-C, Foucher C, Rattier S, Taskinen M-R, Steiner G (2005) Fenofibrate reduces progression to microalbuminuria over 3 years in a placebo-controlled study in type 2 diabetes: results from the Diabetes Atherosclerosis Intervention Study (DAIS). Am J Kidney Dis 45:485-493

12. Levey AS, Bosch JP, Lewis JB, Greene T, Rogers N, Roth D (1999) A more accurate method to estimate glomerular filtration rate from serum creatinine: a new prediction equation. Ann Intern Med 130:461-470

13. Chudleigh RA, Dunseath G, Evans W et al (2007) How reliable is estimation of glomerular filtration rate at diagnosis of type 2 diabetes? Diab Care 30:300-305

14. United States Renal Data System 2009 ADR Atlas (2009) Available from www.usrds.org, accessed 20 December 2009

15. Gaede P, Lund-Andersen H, Parving H-H, Pedersen O (2008) Effect of a multifactorial intervention on mortality in type 2 diabetes. N Engl J Med 358:580-591

16. Ansquer J-C, Dalton RN, Causse E, Crimet D, Malicot KL, Foucher C (2008) Effect of fenofibrate on kidney function: a 6week randomized crossover trial in healthy people. Am J Kidney Dis 51:904-913

17. Rowe JW, Anderes R, Tobin JD, Norris AH, Shock NW (1976) The effect of age on creatinine clearance in men: a cross-sectional and longtitudinal study. J Gerontol 31:155-163

18. Heerspink HL, de Zeeuw D (2010) Composite renal endpoints: was ACCOMPLISH accomplished? Lancet 375:1140-1142

19. Bakris GL, Sarafidis PA, Weir MR et al (2010) Renal outcomes with different fixed-dose combination therapies in patients with hypertension at high risk for cardiovascular events (ACCOMPLISH): a prespecified secondary analysis of a randomised controlled trial. Lancet 375:1173-1181

20. Klahr S, Levey AS, Beck GJ et al (1994) The effects of dietary protein restriction and blood-pressure control on the progression of chronic renal disease. N Engl J Med 330:877-884

21. Forsblom C, Hiukka A, Leinonen E, Sundvall J, Groop PH, Taskinen M-R (2010) Effects of long-term fenofibrate treatment on markers of renal function in type 2 diabetes: FIELD Helsinki Substudy. Diab Care 33:215-220 
22. Sjöström P, Tidman M, Jones I (2005) Determination of the production rate and non-renal clearance of cystatin $\mathrm{C}$ and estimation of the glomerular filtration rate from the serum concentration of cystatin C in humans. Scand J Clin Lab Invest 65:111-124

23. Knight EL, Verhave JC, Spiegelman D et al (2004) Factors influencing serum cystatin $\mathrm{C}$ levels other than renal function and the impact on renal function measurement. Kidney Int 65:14161421

24. Hottelart C, Esper NE, Achard JM, Pruna A, Fournier A (1999) Fenofibrate increases blood creatinine, but does not change the glomerular filtration rate in patients with mild renal insufficiency. Néphrologie 20:41-44

25. Hottelart C, Esper NE, Rose F, Achard J-M, Fournier A (2002) Fenofibrate increases creatinemia by increasing metabolic production of creatinine. Nephron 92:536-541

26. Taal MW, Brenner BM (2000) Renoprotective benefits of RAS inhibition: from ACEI to angiotensin II antagonists. Kidney Int 57:1803-1817

27. Bakris GL, Weir MR (2000) Angiotensin-converting enzyme inhibitor-associated elevations in serum creatinine. Is this a cause for concern? Arch Intern Med 160:685-693

28. Collins R, Armitage J, Parish S, Sleight P, Peto R, for the Heart Protection Study Collaborative Group (2003) MRC/BHF Heart Protection Study of cholesterol-lowering with simvastatin in 5963 people with diabetes: a randomised placebo-controlled trial. Lancet 361:2005-2016

29. Colhoun HM, Betteridge DJ, Durrington PN et al (2009) Effects of atorvastatin on kidney outcomes and cardiovascular disease in patients with diabetes: an analysis from the Collaborative Atorvastatin Diabetes Study (CARDS). Am J Kidney Dis 54:810-819

30. Shepherd J, Kastelein JJP, Bittner V et al (2007) Effect of intensive lipid lowering with atorvastatin on renal function in patients with coronary heart disease: the Treating to New Targets (TNT) Study. Clin J Am Soc Nephrol 2:1131-1139

31. Han SH, Quon MJ, Koh KK (2005) Beneficial vascular and metabolic effects of peroxisome proliferator-activated receptor[alpha] activators. Hypertension 46:1086-1092

32. Anami Y, Kobori S, Sakai M et al (1997) Human beta-migrating very low density lipoprotein induces foam cell formation in human mesangial cells. Atherosclerosis 135:225-234
33. Olufadi R, Byrne CD (2006) Effects of VLDL and remnant particles on platelets. Pathophysiol Haemost Thromb 35:281-291

34. Shen GX (2003) Impact and mechanism for oxidized and glycated lipoproteins on generation of fibrinolytic regulators from vascular endothelial cells. Mol Cell Biochem 246:69-74

35. Muntner P, Coresh J, Smith JC, Eckfeldt J, Klag MJ (2000) Plasma lipids and risk of developing renal dysfunction: the Atherosclerosis Risk in Communities Study. Kidney Int 58:293301

36. Lopes-Virella MF, Carter RE, Gilbert GE et al (2008) Risk factors related to inflammation and endothelial dysfunction in the DCCT/ EDIC cohort and their relationship with nephropathy and macrovascular complications. Diab Care 31:2006-2012

37. Barter PJ, Nicholls S, Rye K, Anantharamaiah GM, Navab M, Fogelman AM (2004) Antiinflammatory properties of HDL. Circ Res 95:764-772

38. Agrawal V, Shah A, Rice C, Franklin BA, McCullough PA (2009) Impact of treating the metabolic syndrome on chronic kidney disease. Nat Rev Nephrol 5:520-528

39. Parving H-H, Lehnert H, Brochner-Mortensen J et al (2001) The effect of irbesartan on the development of diabetic nephropathy in patients with type 2 diabetes. N Engl J Med 345:870-878

40. Retnakaran R, Cull CA, Thorne KI, Adler AI, Holman RR, for the UKPDS Study Group (2006) Risk factors for renal dysfunction in type 2 diabetes. UKPDS 74. Diabetes 55:1832-1839

41. Gerstein HC, Yusuf S, Mann JFE et al (2000) Effects of ramipril on cardiovascular and microvascular outcomes in people with diabetes mellitus: results of the HOPE study and MICRO-HOPE Substudy. Lancet 355:253-259

42. Davis WA, Knuiman MW, Davis TME (2010) An Australian cardiovascular risk equation for type 2 diabetes: the Fremantle Diabetes Study. Intern Med J 40:286-292

43. Toffaletti JG, McDonnell EH (2008) Variation of serum creatinine, cystatin $\mathrm{C}$, and creatinine clearance tests in persons with normal renal function. Clin Chim Acta 395:115-119

44. Levey AS, Stevens LA, Schmid CH et al (2009) A new equation to estimate glomerular filtration Rate. Ann Intern Med 150:604612

45. Warram J, Gearin G, Laffel L, Krolewski A (1996) Effect of duration of type I diabetes on the prevalence of stages of diabetic nephropathy defined by urinary albumin/creatinine ratio. J Am Soc Nephrol 7:930-937 